\title{
Fog Computing-Assisted Energy-Efficient Resource Allocation for High-Mobility MIMO-OFDMA Networks
}

\author{
Lingyun Lu, ${ }^{1}$ Tian Wang $\mathbb{D},{ }^{1}$ Wei Ni, ${ }^{2}$ Kai Li, ${ }^{3}$ and Bo Gao ${ }^{1}$ \\ ${ }^{1}$ College of Computer Science, Beijing Jiaotong University, Beijing, China \\ ${ }^{2}$ Cyber-Physical System, Data61, CSIRO, Australia \\ ${ }^{3}$ Real-Time and Embedded Computing Systems Research Centre (CISTER), 4249015 Porto, Portugal
}

Correspondence should be addressed to Tian Wang; 17120423@bjtu.edu.cn

Received 3 May 2018; Accepted 27 June 2018; Published 11 July 2018

Academic Editor: Fuhong Lin

Copyright (C) 2018 Lingyun Lu et al. This is an open access article distributed under the Creative Commons Attribution License, which permits unrestricted use, distribution, and reproduction in any medium, provided the original work is properly cited.

\begin{abstract}
This paper presents a suboptimal approach for resource allocation of massive MIMO-OFDMA systems for high-speed train (HST) applications. An optimization problem is formulated to alleviate the severe Doppler effect and maximize the energy efficiency (EE) of the system. We propose to decouple the problem between the allocations of antennas, subcarriers, and transmit powers and solve the problem by carrying out the allocations separately and iteratively in an alternating manner. Fast convergence can be achieved for the proposed approach within only several iterations. Simulation results show that the proposed algorithm is superior to existing techniques in terms of system EE and throughput in different system configurations of HST applications.
\end{abstract}

\section{Introduction}

Recent development and deployment of high-speed trains (HSTs) have dramatically improved the efficiency and user experience in interstate transportations. However, providing high data rates and good quality of service (QoS) to passengers in the presence of rapidly varying channel conditions and scarce bandwidth availability is a challenging task [1]. Critical challenges have arisen from real-time communications between HSTs and fixed base stations (BS). Existing narrow-band railway communication systems, such as GSM$\mathrm{R}$, are not suitable for HSTs due to typically low capacity. $5 \mathrm{G}$ technology is currently adopting a so-called network densification approach, which involves the deployment of a large number of base stations (BSs), to increase the network coverage and provide higher throughput to the users [2]. Orthogonal-Frequency Division-Multiple-Access (OFDMA) has been extensively adopted for wideband communications, but severe Doppler shift exists in the communication process because of high mobility, resulting in the difficulties in channel estimation [3] and subsequently destructive intercarrier interference (ICI) [4]. On the other hand, increasing the number of antennas at both transmitters and receivers, also known as Multiple-Input Multiple-Output (MIMO), can improve robustness against ICI. Particularly, MIMO with a large number of antennas has been increasingly studied for enhancing quality and reliability of wideband wireless communications. Unfortunately, the benefits do not come for free. Energy consumption would grow substantially, as the number of antennas increases. An energy-efficient resource allocation of MIMO-OFDMA is expected to balance spectral efficiency and energy efficiency (EE) [5].

There has been a lot of work on wireless resource allocation in static and low-speed mobile system. In [6], it was revealed that network energy can be saved by assigning nonoverlapping frequency bands to different cells. In [7], a power loading algorithm was proposed to maximize the $\mathrm{EE}$ of MIMO. In [8], the authors investigated the energy-efficient bandwidth allocation in downlink flat fading OFDMA channels and maximized the numbers of bits transmitted per joule, by using the Lagrangian and time-sharing techniques. In [9], the authors proposed a hybrid structure of resource allocation in OFDMA cellular systems, which maximized both the EE and the downlink system capacity. The proposed structure, combined with resource allocation, was shown to improve the EE and the system capacity of OFDMA. In [10], 
the resource allocation for energy-efficient OFDMA systems was formulated as a mixed nonconvex and combinatorial optimization problem and solved by exploiting fractional programming. In [11], the energy-efficient configuration of spatial and frequency resources was studied to maximize the EE for downlink MIMO-OFDMA systems in the absence of channel state information (CSI) at the BS. However, none of the existing works have taken into account the destructive ICI. For HSTs at a speed of over $500 \mathrm{~km} / \mathrm{h}$, the fast timevarying channel and the severe Doppler shift have yet to be addressed, and high-mobility communication shall be one of the most important and extreme use scenarios in future 5 th generation (5G) mobile communication networks [1216]. The OFDMA resource allocation strategy was designed for fast-changing mobile environments in [17], where a suboptimal allocation policy was developed at a significant cost of computational complexity.

Fog computing, also known as fogging, is an architecture that uses edge devices to carry out a substantial amount of local computation, storage, and communication [18-20]. We use a fog server at the BS to concentrate data, data processing, and applications. The fog server can increase overall computing capability, which helps in efficient resource allocation and utilization.

Fog computing emphasizes proximity to end-users and client objectives, dense geographical distribution and local resource pooling, latency reduction, and backbone bandwidth savings. Therefore, we use this technology to provide practical value for real-time implementation of HST communications.

This paper aims to design an efficient resource allocation strategy to improve the communication performance of HSTs. After analyzing the multiuser MIMO-OFDMA downlink system, the influence of mobility on the system is quantified. A mathematical model is put forth to maximize the EE of the system. To tackle the problem, an iterative algorithm with fast convergence is proposed. Specifically, we propose to decouple the problem between the allocations of antennas, subcarriers, and transmit powers and solve the problem by carrying out the allocations separately and iteratively in an alternating manner. Fast convergence can be achieved for the proposed approach within only several iterations. Simulation results demonstrate the gain of the proposed approach in terms of EE and throughput, as compared with existing schemes.

The rest of the paper is organized as follows. We present the system model in Section 2 and formulate and solve the problem of interest in Section 3. In Section 4, the simulation results are provided, followed by conclusions in Section 5 .

\section{System Model}

The system of interest is a multiuser MIMO-OFDMA system, as illustrated in Figure 1, where there is a fixed BS equipped with $M$ transmit antennas $(M \gg 1)$ and $K$ user terminals located in a HST. A fog server is employed at the BS to help the resource allocation computation. Each of the user equipment has a single receive antenna. The users share radio resources for down services. Each of the user equipment has a single

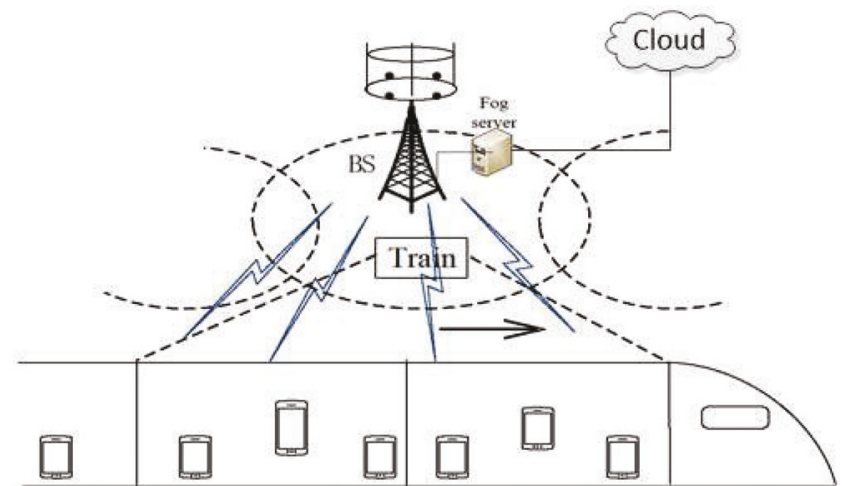

FIGURE 1: Networks architecture for multiuser MIMO system.

receive antenna. The users share radio resources for down services. Different users are assigned with different OFDM subcarriers and different antennas, given the large number of transmit antennas. Coherent beamforming is carried out at the BS to produce physical beams towards the users.

The speed of HST can lead to severe Doppler shifts. Let $h_{k, n}$ denote the complex channel gain between the BS and user $k$ on subcarrier $n$. The total number of subcarriers is $N$. The knowledge on $h_{k, n}$ can be inaccurate at the BS, because of the fast-changing HST environment and hence estimation errors. We assume

$$
h_{k, n}=\widehat{h}_{k, n}+\Delta h_{k, n}
$$

where $\widehat{h}_{k, n}$ is the estimate of $h_{k, n}$ at the BS and $\Delta h_{k, n}$ is an independent and identically distributed (i.i.d.) measurement error. $\Delta h_{k, n}$ yields a complex Gaussian distribution due to the use of the Minimum Mean Square Error (MMSE) estimators. $\Delta h_{k, n} \sim N\left(\mu, \sigma_{e}^{2}\right)$ and

$$
\sigma_{e}^{2}=\frac{1}{1+\left(\Delta f / f_{d}\right)\left(p_{k, n} / n_{0}\right)}
$$

where $\Delta f$ is the subcarrier interval and $f_{d}$ is the maximum Doppler shift which can be written as $f_{d}=V \cdot f_{c} / c$. $c$ is the speed of light. $f_{c}$ is the carrier frequency. $p_{k, n}$ is the transmit power allocated to user $k$ on subcarrier $n$. $n_{0}$ is the noise power spectral density [10].

We assume that each subcarrier has an equal bandwidth of $B$. Therefore, the total bandwidth of the system is $B_{\text {tot }}=$ $N B$. We also assume that each subcarrier is assigned an equal transmit power; i.e., $p_{k, n}=P_{k} / b_{k}=p_{k}$, where $b_{k}$ and $P_{k}$ are the number of subcarriers and the transmit power of the BS allocated to user $k$, respectively.

The Doppler shift can compromise the orthogonality between OFDM subcarriers, resulting in ICI [22]. At a speed of $V$, the power of ICI on a subcarrier can be written as [23]

$$
I C I(V)=\sum_{n=1}^{N} \frac{\left(T_{s} f_{d}\right)^{2}}{2} \sum_{j=1, j \neq n}^{N} \frac{1}{(j-n)^{2}}
$$

where $T_{S}$ denotes the duration of an OFDM symbol. 
In the case that $M \longrightarrow \infty$, the receive signal-to-noise ratio (SNR) can be approximated to [17]

$$
\rho_{k, n} \approx \frac{p_{k, n} l_{k} M_{k}\left(1-\sigma_{e}^{2}\right)}{n_{0} B+p_{k} I C I(V)}
$$

where $M_{k}$ is the number of antennas of the BS assigned to user $k$.

The asymptotic rate of the MIMO can be achieved based on the random matrix theory [17]. Specifically, the rate asymptotically converges to the average rate in mean square. The asymptotic rate can be replaced with the average data rate. The total data rate of user $k$ converges to

$$
r_{k}=b_{k} B \log _{2}\left(1+\frac{p_{k} l_{k} M_{k}\left(1-\sigma_{e}^{2}\right)}{n_{0} B+p_{k} I C I(V)}\right) .
$$

We also consider nonideal circuit power at the BS. We can adopt a linear model [24] at the BS to characterize the circuit power consumption, as given by

$$
\phi=P_{c} \max _{k}\left\{M_{k}\right\}+\sum_{k=1}^{K} b_{k} p_{k}+P_{0}
$$

where $P_{c}$ is the power consumption per active antenna, consisting of the power consumption of filtering, mixing, power amplification, and digital-to-analog conversion. $P_{0}$ is the constant part of the power consumption at the BS and is independent of the number of active antennas.

\section{Optimization Problem Formulation}

The goal of this paper is to maximize the EE of the BS, which can be formulated as

$$
\begin{array}{ll}
\max _{M B P} & \left\{Q(\boldsymbol{M}, \boldsymbol{B}, \boldsymbol{P})=\frac{R(\boldsymbol{M}, \boldsymbol{B}, \boldsymbol{P})}{\phi(\boldsymbol{M}, \boldsymbol{B}, \boldsymbol{P})}\right\} \\
\text { s.t. } \quad & C 1: \sum_{k=1}^{K} P_{k} \leq P_{T}, \\
& C 2: r_{k} \geq R_{\min }, \\
& C 3: \sum_{k}^{K} b_{k} \leq N, \\
& C 4: M_{k} \leq M
\end{array}
$$

where, given (5) and (6), the EE of the BS can be written as

$$
\begin{aligned}
Q & =\frac{R}{\phi} \\
& =\frac{\sum_{k=1}^{K} b_{k} B \log _{2}\left(1+p_{k} l_{k} M_{k}\left(1-\sigma_{e}^{2}\right) /\left(n_{0} B+p_{k} I C I_{n}(V)\right)\right)}{P_{c} \max _{k}\left\{M_{k}\right\}+\sum_{k=1}^{K} b_{k} p_{k}+P_{0}} ;
\end{aligned}
$$

the vector $\boldsymbol{B}=\left[b_{1}, b_{2}, \ldots, b_{K}\right]^{\mathrm{T}}$ collects the subcarrier allocation of all $K$ users; $\boldsymbol{M}=\left[M_{1}, M_{2}, \ldots, M_{K}\right]^{\mathrm{T}}$ collects the antenna allocations for the users; and $\boldsymbol{P}=\left[P_{1}, P_{2}, \ldots, P_{K}\right]^{\mathrm{T}}$ collects the power allocations of the users. The constraint $\mathrm{Cl}$ specifies the total transmit power constraint $P_{T}$. C2 specifies the minimum data rate per user. C3 and C4 restrict the total numbers of subcarriers and antennas, respectively.

Clearly, problem (7) is a combinatorial mixed integer programming problem. The objective of (7) also has a fractional form with variables in the denominator of the objective. All this makes (7) a NP-hard nonconvex problem with poor tractability. In order to solve the problem efficiently, we develop a suboptimal solution, where the subcarriers allocation, antennas, and transmit powers are optimized separately and sequentially in an alternating manner.

3.1. Subcarrier Allocation. Given $M$ and $P$, we first propose to allocate subcarriers to maximize the $\mathrm{EE}$ while satisfying the minimum data rates of the users. According to the objective of (8), the subcarrier allocation can be expressed as

$$
b_{k}=\arg \max _{\boldsymbol{B}} Q(\boldsymbol{M}, \boldsymbol{B}, \boldsymbol{P}) .
$$

We propose to allocate subcarriers based on the criterion of EE. First, we calculate the number of subcarriers allocated to each user according to the minimum data rate of the user. Then, we choose the user with the highest EE and allocate a subcarrier to the user, one user after another, and this repeats until all users are allocated or all subcarriers are assigned. The proposed allocation of subcarriers can be summarized in Algorithm 1.

3.2. Transmit Power and Antenna Allocation. Given the subcarrier allocation developed in Section 3.1, problem (7) can be reformulated to a fractional programing problem with respect to $M$ and $P$, as given by [25]

$$
\begin{aligned}
F(q)=\max & \{R(\boldsymbol{M}, \boldsymbol{P})-q \phi(\boldsymbol{M}, \boldsymbol{P})\} \\
\text { s.t. } & \mathrm{C} 1, \mathrm{C} 2, \mathrm{C} 4 .
\end{aligned}
$$

This is mixed integer programming. We proceed to relax the integer constraint C4, i.e., $M_{k}$ to $\widetilde{M}_{k} \in\left[M_{\min }, M\right] . M_{\min }$ is the minimum number of antennas to meet the requirements of uninterrupted transmission for all users [10]. As a result, (10) can be further reformulated as

$$
\begin{array}{ll}
\max & \{R(\boldsymbol{M}, \boldsymbol{P})-q \widetilde{\phi}(\boldsymbol{M}, \boldsymbol{P})\} \\
\text { s.t. } & C 1: \sum_{k=1}^{K} P_{k} \leq P_{T}, \\
& C 2: r_{k} \geq R_{\min }, \\
& C 4: \widetilde{M}_{k} \in\left[M_{\mathrm{min}}, M\right]
\end{array}
$$

where $q$ is the optimal solution for problem (10). 


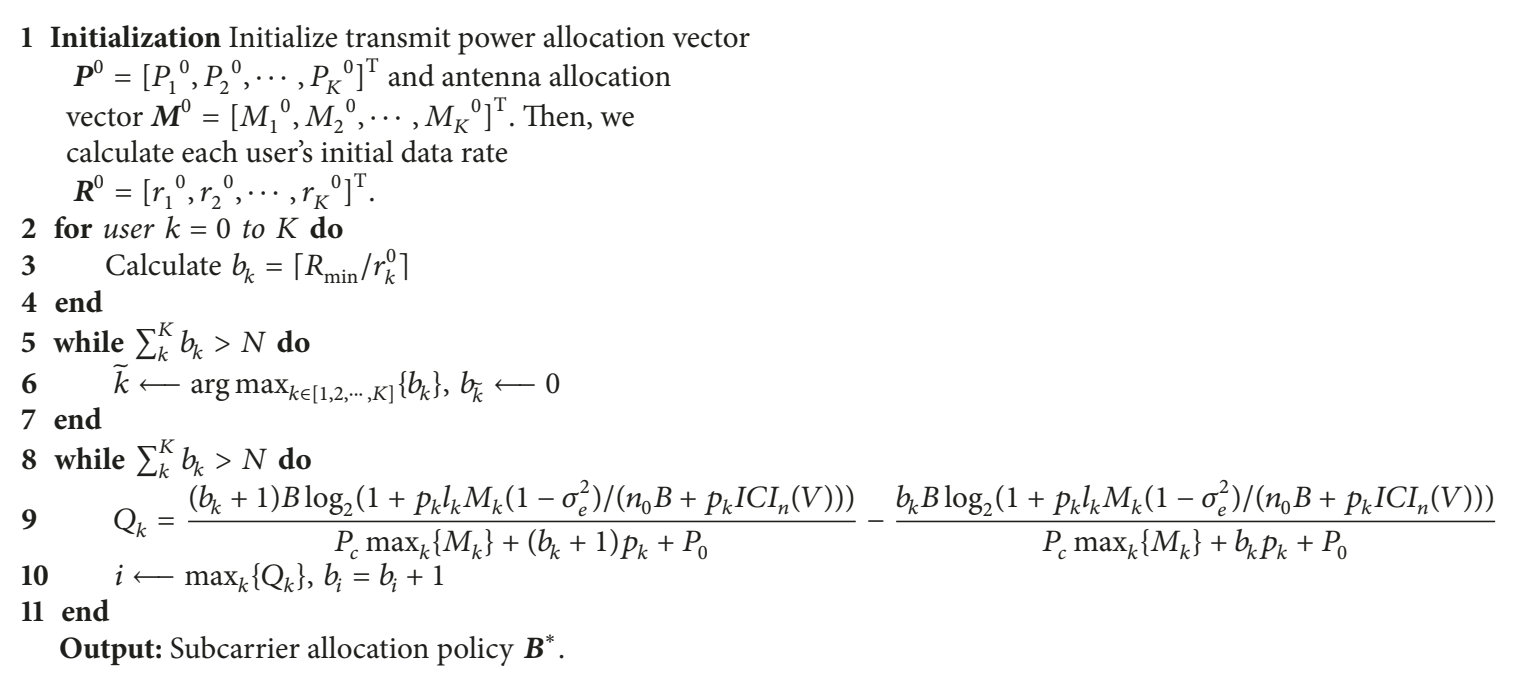

Algorithm 1

We can prove that (11) is a concave function by evaluating the Hessian matrix of $-F(q)$, as given by

$$
\left[\begin{array}{cc}
\frac{a^{2} b P_{k}^{2}}{\ln 2\left(n_{0} b+c P_{k}+a P_{k} M_{k}\right)^{2}} & -\frac{a b^{2} n_{0}}{\ln 2\left(n_{0} b+c P_{k}+a P_{k} M_{k}\right)^{2}} \\
-\frac{a b^{2} n_{0}}{\ln 2\left(n_{0} b+c P_{k}+a P_{k} M_{k}\right)^{2}} & \frac{a b^{2} n_{0} M_{k}\left(2 b c n_{0}+a b n_{0} M_{k}+2 c^{2} P_{k}+2 a c P_{k} M_{k}\right)}{\ln 2\left(n_{0} b+c P_{k}+a P_{k} M_{k}\right)^{2}\left(n_{0} b+c P_{k}\right)^{2}}
\end{array}\right]
$$

where $a=l_{k}\left(1-\sigma_{e}^{2}\right), b=B b_{k}$, and $c=I C I(V)$. Both the determinant of the Hessian matrix and its $k$ th order principal matrix are nonnegative. Thus the Hessian matrix is positive semidefinite. Hence, $-F(q)$ is strictly convex. As a result, the objective function of problem (11) is jointly concave over $(\boldsymbol{M}, \boldsymbol{P})$ while all the constraints are linear. In addition, (11) yields the Slater conditions [26] and therefore holds strong duality. The dual problem of (11) and the primary problem (11) have zero duality gap.

Given $q$, the Lagrangian function can be written as

$$
\begin{aligned}
& L(\boldsymbol{M}, \boldsymbol{P}, \boldsymbol{\lambda}, \mu) \\
& =\mu\left(P_{T}-\sum_{k=1}^{K} P_{k}\right) \\
& \quad+\sum_{k=1}^{K}\left(1+\lambda_{k}\right) b_{k} B \log _{2}\left(1+\frac{p_{k} l_{k} M_{k}\left(1-\sigma_{e}^{2}\right)}{n_{0} B+p_{k} I C I_{n}(V)}\right) \\
& \quad-\sum_{k=1}^{K} \lambda_{k} R_{\min }-q\left[P_{c} \max _{k}\left\{M_{k}\right\}+\sum_{k=1}^{K} b_{k} p_{k}+P_{0}\right],
\end{aligned}
$$

where $\lambda$ collects the Lagrange multipliers associated with constraint C2 and $\lambda_{k} \geq 0 ; \mu \geq 0$ is the Lagrange multiplier associated with constraint C1. The dual problem of (11) is given by

$$
\min _{\boldsymbol{\lambda}, \mu \geq 0}\left\{\max _{\boldsymbol{M}, \boldsymbol{P}}\{L(\boldsymbol{M}, \boldsymbol{P}, \boldsymbol{\lambda}, \mu)\}\right\}
$$

Given $(\lambda, \mu)$, according to the KKT conditions, the optimal power allocation, denoted by $\boldsymbol{P}^{*}$, and antenna allocation, denoted by $\boldsymbol{M}^{*}$, can be obtained as

$$
\begin{aligned}
P_{k}^{*} & =\frac{b \sqrt{\left(a^{2} n_{0} d+4 a^{2} c+4 a c^{2}\right) / n_{0} d}-a-2 c}{2 a c+2 a c^{2}} ; \\
M_{k}^{*} & =\left[\frac{b_{k} B\left(1+\lambda_{k}\right)}{q P_{c} \ln (2)}-\frac{1}{\alpha_{M}(V)}\right\rceil,
\end{aligned}
$$

where $a=l_{k} M_{k}\left(1-\sigma_{e}^{2}\right), b=n_{0} B b_{k}, c=I C I(V), d=(q+$ $\mu) \ln 2 /\left(1+\lambda_{k}\right)$, and $\alpha_{M}(V)=P_{k} l_{k}\left(1-\sigma_{e}^{2}\right) /\left(n_{0} B+P_{k} I C I(V)\right)$.

The subgradient method can be employed to obtain $(\lambda, \mu)$ in an interactive manner, as given by

$$
\begin{aligned}
\mu(t+1) & =\left[\mu(t)-\delta_{1}(t) L^{\prime}(\mu(t))\right]^{+} ; \\
\lambda_{k}(t+1) & =\left[\lambda_{k}(t)-\delta_{2}(t) L^{\prime}\left(\lambda_{k}(t)\right)\right]^{+},
\end{aligned}
$$




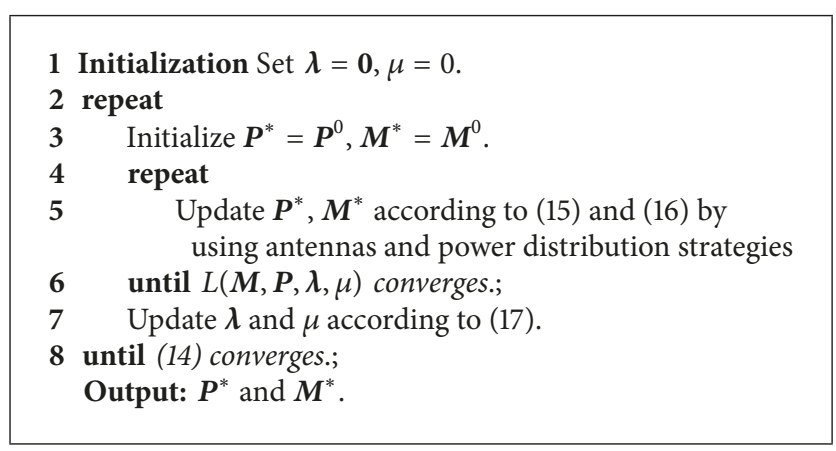

Algorithm 2

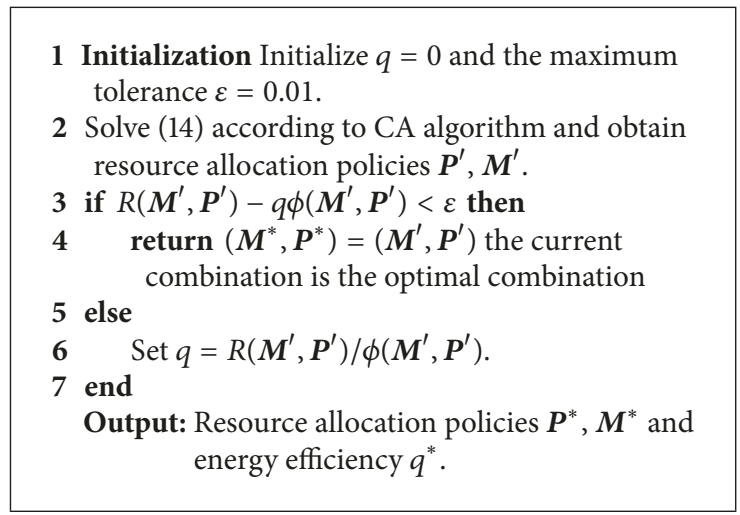

Algorithm 3

where $[x]^{+}=\max \{0, x\} ; t \geq 0$ is the index for the iterations. $\delta_{1}(t)>0$ and $\delta_{2}(t)>0$ are the step sizes to adjust $\mu(t)$ and $\lambda k(t)$, respectively; and $L^{\prime}(\mu(t))$ and $L^{\prime}\left(\lambda_{k}(t)\right)$ are the subgradients of the Lagrangian function at $\mu(t)$ and $\lambda_{k}(t)$, respectively.

The resource allocation policy can be developed based on (15)-(17). Since $(\lambda, \mu)$ and $(M, P)$ can be decoupled in (15), (16), and (17), we can use an improved coordinate ascent (CA) method, where, during each iteration, we first optimize $(M, P)$, given $(\lambda, \mu)$ and $q$, and then optimize $(\lambda, \mu)$, given $(M, P)$, in an alternating fashion until convergence. Given $q$, the proposed allocation of transmit antennas and subcarriers is summarized in Algorithm 2.

Finally, we can use the Dinkelbach method [25] to update q. The solution for problem (11) can be summarized in Algorithm 3.

\section{Simulation Results}

In this section, we simulate the proposed algorithm to verify its effectiveness, where block Rayleigh fading channels are considered. Other simulation parameters are listed in Table 1. We note that the proposed algorithm can be applied under any channel conditions, such as Rician fading channels.

For comparison purpose, the following two resource allocation schemes are also stimulated.

(1) Band allocation based on SNR (BABS) algorithm [27]: it is used for subcarrier allocation. The transmit powers and
TABLE 1: Simulation parameters.

\begin{tabular}{lc}
\hline Parameter Notation & Value \\
\hline Speed of electromagnetic wave $c$ & $3 \times 108 \mathrm{~m} / \mathrm{s}$ \\
Noise power spectral density $n_{0}$ & $2 \times 10^{-7} \mathrm{~W} / \mathrm{Hz}$ \\
Minimum data rate & $3.0 \times 10^{7} \mathrm{bit} / \mathrm{s}$ \\
Center carrier frequency $f_{c}$ & $2.6 \mathrm{GHz}$ \\
Subcarriers number $N$ & 64 \\
Total Bandwidth $B_{\text {tot }}$ & $5 \mathrm{MHz}$ \\
Power consumption per antenna $P_{c}$ & $30 \mathrm{dBm}[21]$ \\
Static power consumption $P_{0}$ & $40 \mathrm{dBm}[21]$ \\
Minimum antenna $M_{\min }$ & $24[8]$ \\
Maximum antenna $M \max$ & 100 \\
\hline
\end{tabular}

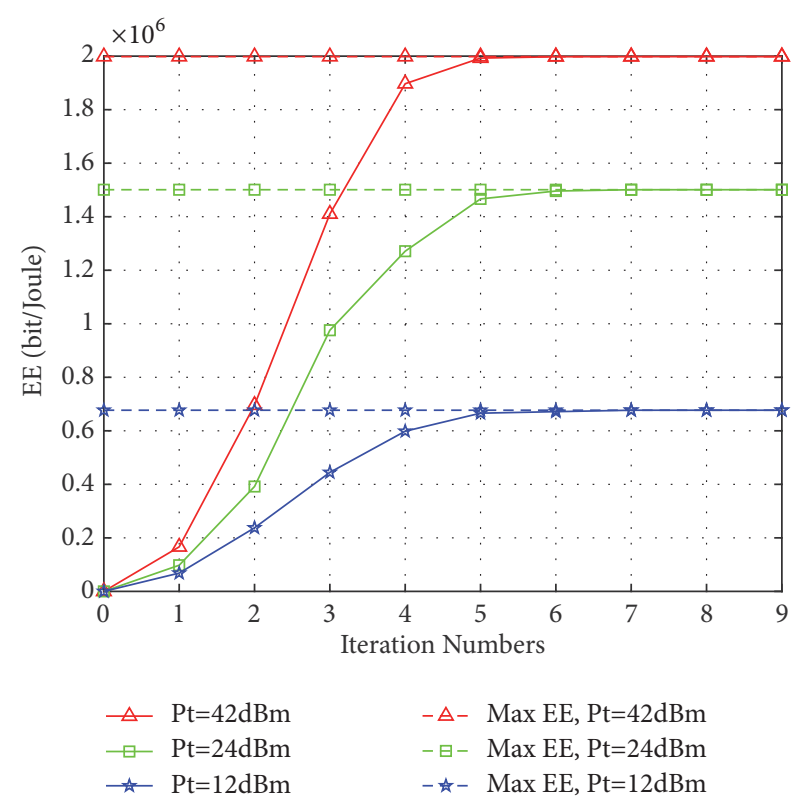

FIGURE 2: System EE versus iteration numbers for different transmit power with $K=20, N=64, V=500 \mathrm{~km} / \mathrm{h}$.

antennas are allocated in the same way as in the proposed algorithm.

(2) EMMPA algorithm [28]: this algorithm first allocates subcarriers evenly and then allocates the rest of subcarriers to the users with the best channel condition. The scheme developed in [26] is used for the transmit power and antenna allocation.

Figure 2 shows the convergence of the proposed algorithm with different transmit powers, where $K=20, N=64$, and $V=500 \mathrm{~km} / \mathrm{h}$. It is seen that the EE of the proposed algorithm increases and quickly stabilizes with the growth of iterations. The maximum of the EE can be attained after around only six iterations.

Figure 3 plots the system EE versus the maximum transmit power, where $K=20$ and $V=500 \mathrm{~km} / \mathrm{h}$. We can see that the system EE increases with maximum transmit power. When the transmit power is large enough, the system EE stabilizes. This is because the BS does not need to activate extra antennas or consume extra power when the 


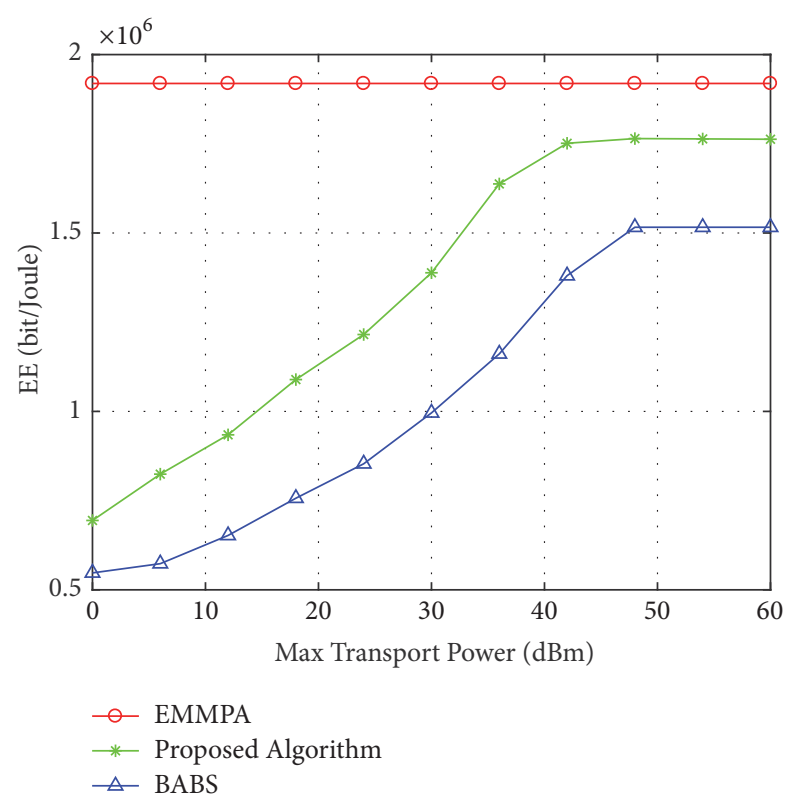

FIgURE 3: System EE versus maximum transmit power with $K=20$, $V=500 \mathrm{~km} / \mathrm{h}$.

system maximum EE is reached. The figure also shows that our proposed algorithm performs between the BABS and EMMPA algorithms. The system EE of EMMPA is higher than our proposed algorithm since EMMPA does not have the constraint of $P_{T}$ and, thus, has a fixed EE value. Additionally, EMMPA is an unconstrained problem to maximize the system EE. The system EE of our proposed algorithm is higher than that of the BABS algorithm because our approach is based on the maximization of EE, while the BABS is based on the minimization of SNR.

Figure 4 presents the system throughput versus the moving speed $V$, where $P_{T}=40 \mathrm{dBm}$ and $K=20$. We can see that, as $V$ increases, the system throughput significantly decreases. This is because ICI power and channel estimation error are increasingly severe and thus increasingly detrimental to communication quality. The system throughput is about $20.7 \%$ higher under our proposed algorithm than under BABS algorithm. EMMPA provides the lowest throughput because of its nature of an unconstrained optimization of maximizing EE without constraints. BABS is to minimize the transmit power while allocating subcarriers. The conclusion drawn is that our proposed algorithm can significantly improve throughput.

Figure 5 shows the system EE versus the number of users $K$, where $P_{T}=40 \mathrm{dBm}$ and $V=500 \mathrm{~km} / \mathrm{h}$. It can be seen that the system EE decreases with the number of users. This is because when the number of subcarriers is fixed, each user can be allocated with a less number of subcarriers, resulting in an increase of the transmit powers to satisfy the users data rate requirement. EMMPA has no demand for the data rate, but the subcarriers assigned to the users who have better channel conditions decrease, and thus system EE also decreases.

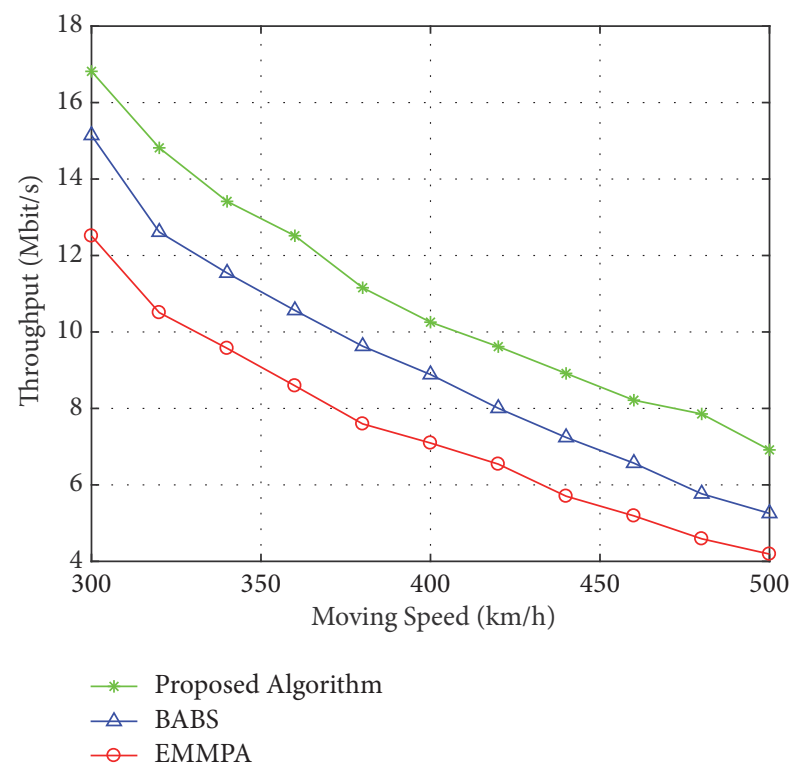

FIgURE 4: System throughput versus the moving speed with $P_{T}=$ $40 \mathrm{dBm}, K=20$.

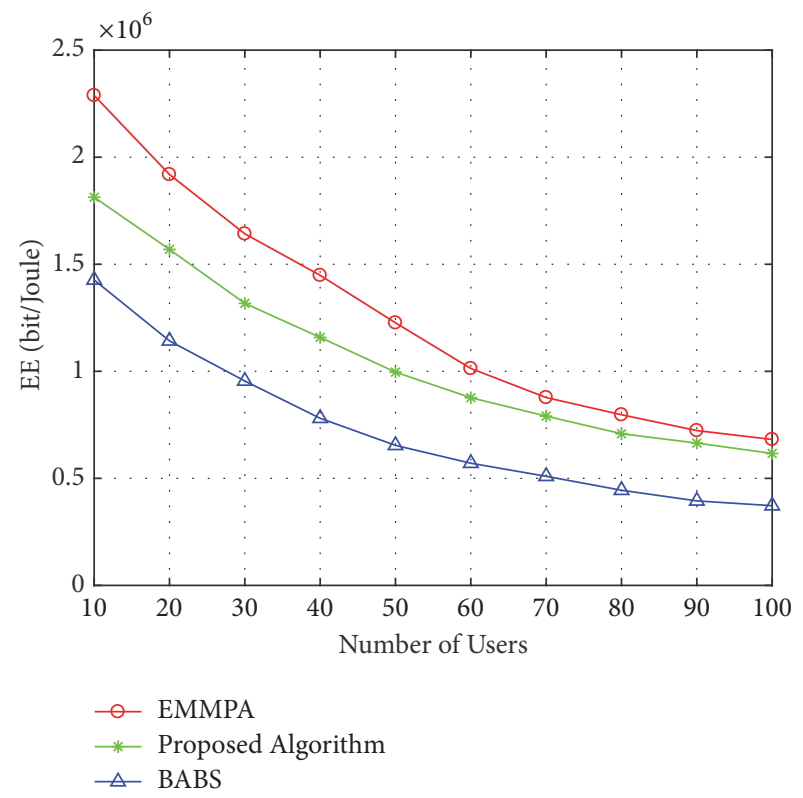

FIgURE 5: System EE versus number of users with $P_{T}=40 \mathrm{dBm}$, $V=500 \mathrm{~km} / \mathrm{h}$.

\section{Conclusion}

This paper models the resource allocation strategy for multiuser MIMO-OFDMA downlink system for HSTs, where subcarriers, transmit power, and antennas are jointly optimized. Specifically, we propose an iterative suboptimal algorithm to optimize the system EE with fast convergence. In terms of the system performance, simulation results show 
that both EE and throughput are improved. Furthermore, the proposed approach is able to fast stabilize within only several iterations and therefore provides practical value for real-time implementation of HST communications.

\section{Data Availability}

The data used to support the findings of this study are available from the corresponding author upon request. No additional data are available.

\section{Conflicts of Interest}

The authors declare that they have no conflicts of interest.

\section{Acknowledgments}

This project is supported by the National Natural Science Foundation of China (Grant no. 61771002).

\section{References}

[1] A. O. Laiyemo, P. Luoto, P. Pirinen, and M. Latva-Aho, "Feasibility studies on the use of higher frequency bands and beamforming selection scheme for high speed train communication," Wireless Communications and Mobile Computing, vol. 2017, 2017.

[2] D. Thembelihle, M. Rossi, and D. Munaretto, "Softwarization of Mobile Network Functions towards Agile and Energy Efficient 5G Architectures: A Survey," Wireless Communications and Mobile Computing, vol. 2017, 2017.

[3] C. Zhang, P. Fan, Y. Dong, and K. Xiong, "Service-based highspeed railway base station arrangement," Wireless Communications and Mobile Computing, vol. 15, no. 13, pp. 1681-1694, 2015.

[4] D. Gesbert, S. Hanly, H. Huang, S. Shamai Shitz, O. Simeone, and W. Yu, "Multi-cell MIMO cooperative networks: a new look at interference," IEEE Journal on Selected Areas in Communications, vol. 28, no. 9, pp. 1380-1408, 2010.

[5] I. Chih-Lin, C. Rowell, S. Han, Z. Xu, G. Li, and Z. Pan, "Toward green and soft: a $5 \mathrm{G}$ perspective," IEEE Communications Magazine, vol. 52, no. 2, pp. 66-73, 2014.

[6] O. Holland, V. Friderikos, and A. H. Aghvami, "Green spectrum management for mobile operators," in Proceedings of the 2010 Ieee Globecom Workshops, pp. 1458-1463, Miami, FL, USA, December 2010.

[7] R. S. Prabhu and B. Daneshrad, "Energy-efficient power loading for a MIMO-SVD system and its performance in flat fading," in Proceedings of the 53rd IEEE Global Communications Conference, GLOBECOM 2010, IEEE, Miami, FL, USA, December 2010.

[8] A. Akbari, R. Hoshyar, and R. Tafazolli, "Energy-efficient resource allocation in wireless OFDMA systems," in Proceedings of the IEEE 21st International Symposium on Personal Indoor and Mobile Radio Communications (PIMRC '10), pp. 1731-1735, IEEE, Instanbul, Turkey, September 2010.

[9] X. Xiao, X. Tao, Y. Jia, and J. Lu, "An energy-efficient hybrid structure with resource allocation in OFDMA networks," in Proceedings of the 2011 IEEE Wireless Communications and Networking Conference, WCNC 2011, pp. 1466-1470, Mexico, March 2011.
[10] D. W. K. Ng, E. S. Lo, and R. Schober, "Energy-efficient resource allocation in OFDMA systems with large numbers of base station antennas," IEEE Transactions on Wireless Communications, vol. 11, no. 9, pp. 3292-3304, 2012.

[11] G. Y. Li, Z. Xu, C. Xiong et al., "Energy-efficient wireless communications: tutorial, survey, and open issues," IEEE Wireless Communications Magazine, vol. 18, no. 6, pp. 28-34, 2011.

[12] K. Xiong, P. Fan, Y. Zhang, and K. Ben Letaief, "Towards 5G High Mobility: A Fairness-Adjustable Time-Domain Power Allocation Approach," IEEE Access, vol. 5, pp. 11817-11831, 2017.

[13] Y. Lu, K. Xiong, P. Fan, and Z. Zhong, "Optimal Multicell Coordinated Beamforming for Downlink High-Speed Railway Communications," IEEE Transactions on Vehicular Technology, vol. 66, no. 10, pp. 9603-9608, 2017.

[14] K. Xiong, Y. Zhang, P. Fan, H.-C. Yang, and X. Zhou, "Mobile Service Amount Based Link Scheduling for High-Mobility Cooperative Vehicular Networks," IEEE Transactions on Vehicular Technology, vol. 66, no. 10, pp. 9521-9533, 2017.

[15] T. Li, K. Xiong, P. Fan, and K. B. Letaief, "Service-Oriented Power Allocation for High-Speed Railway Wireless Communications," IEEE Access, vol. 5, pp. 8343-8356, 2017.

[16] K. Xiong, B. Wang, C. Jiang, and K. J. R. Liu, "A Broad Beamforming Approach for High-Mobility Communications," IEEE Transactions on Vehicular Technology, vol. 66, no. 11, pp. 10546-10550, 2017.

[17] R. Couillet and M. Debbah, Random Matrix Methods for Wireless Communications, Cambridge University Press, Cambridge, UK, 2011.

[18] X. Lyu, H. Tian, W. Ni, Y. Zhang, P. Zhang, and R. P. Liu, "Energy-Efficient Admission of Delay-Sensitive Tasks for Mobile Edge Computing," IEEE Transactions on Communications, vol. 66, no. 6, pp. 2603-2616, 2018.

[19] X. Lyu, W. Ni, H. Tian et al., "Optimal schedule of mobile edge computing for internet of things using partial information," IEEE Journal on Selected Areas in Communications, vol. 35, no. 11, pp. 2606-2615, 2017.

[20] X. Lyu, C. Ren, W. Ni, H. Tian, and R. P. Liu, "Distributed Optimization of Collaborative Regions in Large-Scale Inhomogeneous Fog Computing," IEEE Journal on Selected Areas in Communications, vol. 36, no. 3, pp. 574-586, 2018.

[21] T. M. Nguyen, V. N. Ha, and L. Bao Le, "Resource allocation optimization in multi-user multi-cell massive MIMO networks considering pilot contamination," IEEE Access, vol. 3, pp. 1272 1287, 2015.

[22] Y. Zhao, X. Li, Y. Li, and H. Ji, "Resource allocation for highspeed railway downlink MIMO-OFDM system using quantumbehaved particle swarm optimization," in Proceedings of the 2013 IEEE International Conference on Communications, ICC 2013, pp. 2343-2347, Hungary, June 2013.

[23] T. Wang, J. G. Proakis, E. Masry, and J. R. Zeidler, "Performance degradation of OFDM systems due to doppler spreading," IEEE Transactions on Wireless Communications, vol. 5, no. 6, pp. 1422-1432, 2006.

[24] T. M. Nguyen and L. B. Le, "Joint pilot assignment and resource allocation in multicell massive MIMO network: Throughput and energy efficiency maximization," in Proceedings of the 2015 IEEE Wireless Communications and Networking Conference, WCNC 2015, pp. 393-398, IEEE, New Orleans, LA, USA, March 2015.

[25] W. Dinkelbach, “On nonlinear fractional programming," Management Science, vol. 13, no. 7, pp. 492-498, 1967. 
[26] S. Boyd and L. Vandenberghe, Convex Optimization, Cambridge University Press, 2004.

[27] D. Kivanc, G. Li, and H. Liu, "Computationally efficient bandwidth allocation and power control for OFDMA," IEEE Transactions on Wireless Communications, vol. 2, no. 6, pp. 11501158, 2003.

[28] G. Miao, "Energy-efficient uplink multi-user MIMO," IEEE Transactions on Wireless Communications, vol. 12, no. 5, pp. 2302-2313, 2013. 


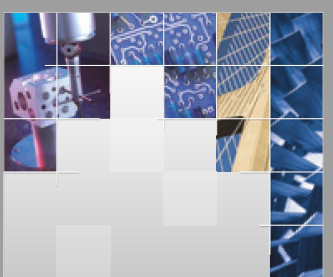

\section{Enfincering}
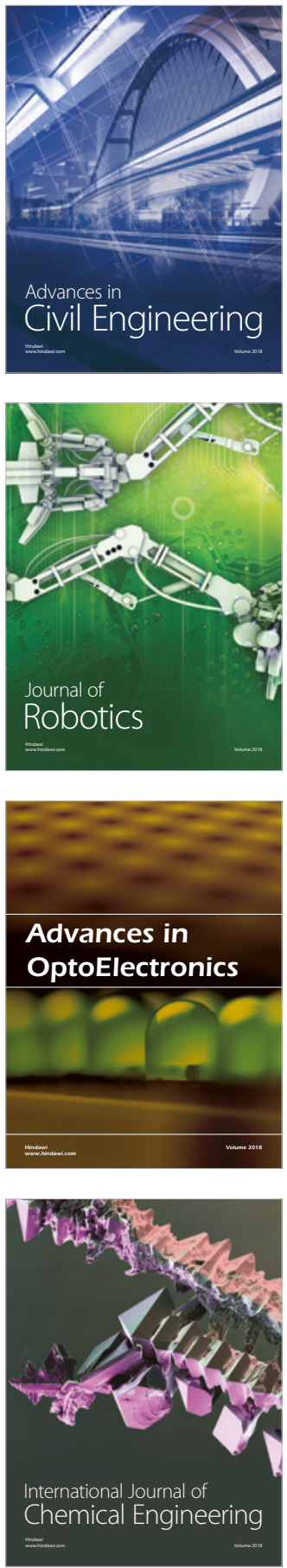

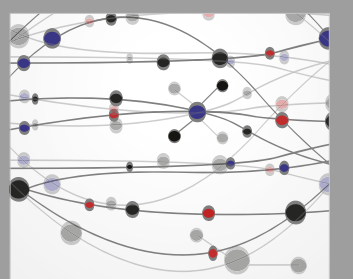

\section{Rotating \\ Machinery}

The Scientific World Journal

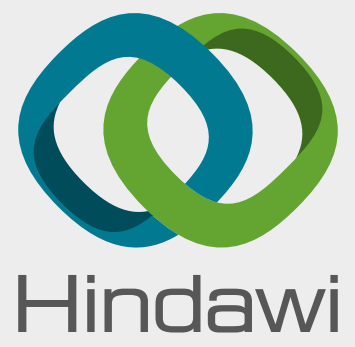

Submit your manuscripts at

www.hindawi.com
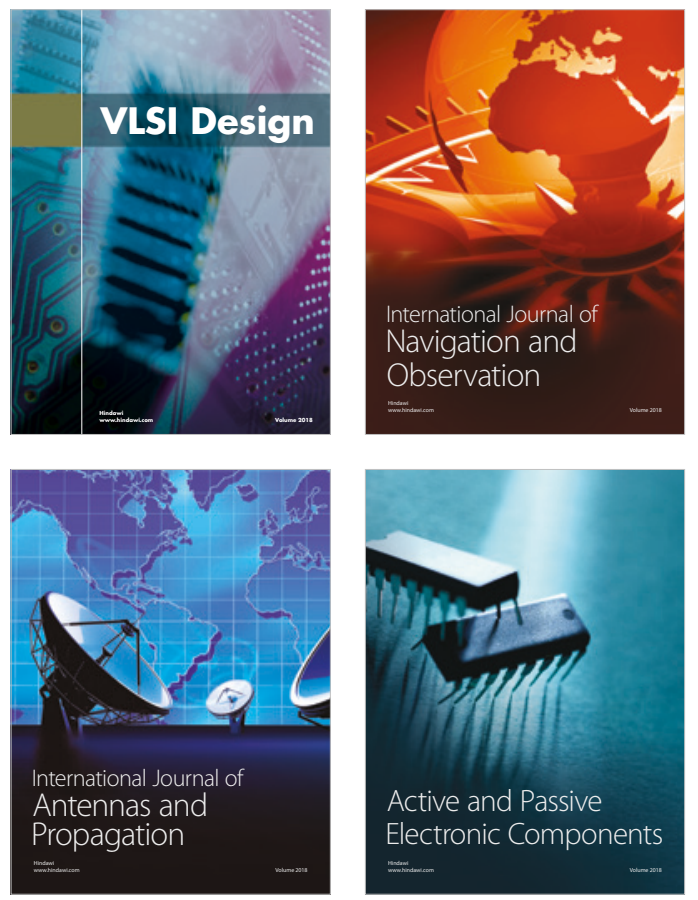
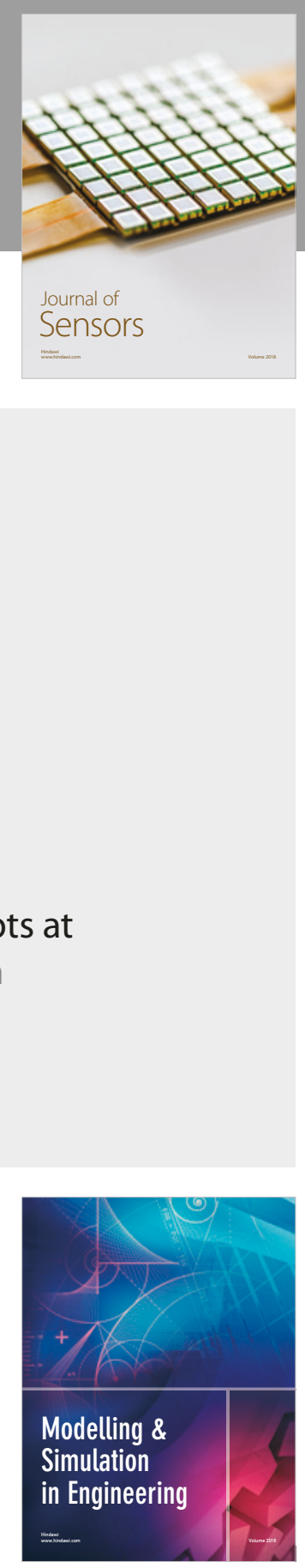

\section{Advances \\ Multimedia}
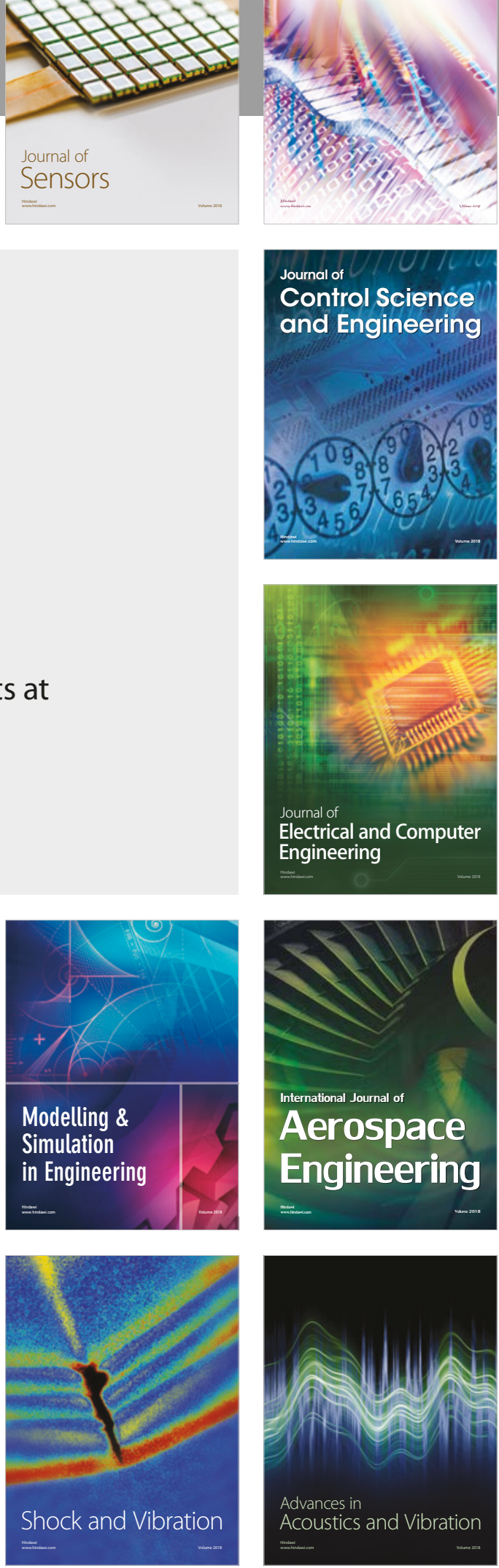